\title{
iVote: A simple system to conduct polls and quiz in class settings
}

\author{
Julien T. Groulx ${ }^{\mathrm{a}}$ and Denis Cousineau ${ }^{\mathrm{a}, \boldsymbol{}}$ \\ ${ }^{a}$ Université d’Ottawa
}

\begin{abstract}
Teaching tools are necessary to promote learning and encourage the understanding of course content. Among those tools, televoting is one by which students can answer anonymously on a question presented by an instructor. Here, we present ivote, a small application that can be used to that end. ivote is free and does not require registration from the professor nor the students, and does not present advertisements. This software can be used in conjunction with PowerPoint with minimal programming; the votes can also be easily exported to SPSS, Excel, Mathematica or R. Students can express their vote as long as they have an internet access either by entering a URL or by scanning a QR code.
\end{abstract}

Keywords $\backsim$ tools, interactive teaching, in-class poll. Tools $\backsim$ PowerPoint, SPSS, R, Python, Mathematica.

Acting Editor $\square$ Gyslain Giguère (Université de Montréal)

Reviewers

- One anonymous reviewer denis.cousineau@uottawa.ca

JTG: 0000-0003-1158-7356; DC: 0000-0001-5908-0402

\section{Introduction}

Technology and software evolve constantly, sometimes to facilitate knowledge acquisition. Teaching tools are useful to instructors because they allow concrete representations of theoretical concepts. Such tools also lead to better communication between instructors and students by reinforcing participation and interest which in turn improves teaching. Conversely, a student's lack of interest or motivation has negative consequences on the general learning experience and on the knowledge acquired.

As one example, statistics classes are often recognized as the most disliked courses taken by social sciences students (Hogg, 1991). Students can be anxious and unmotivated to take part in these classes. This can be related to the expectations of the students towards the class material and their own abilities. It can also be related to inefficient teaching approaches (Vaughn, 2009), which are often geared for mathematically inclined students (Stuart, 1995). Changing the perceptions that students have towards statistics might be a way to reduce anxiety and negative predispositions. Teaching tools are in that case beneficial, as abstract concepts used in statistics such as sampling can be taught with concrete manipulations (Stuart, 1995).

Similar conclusions were drawn by the Guidelines for Assessment and Instruction in Statistics Education's College Report (Aliaga et al., 2005). In fact, the report made multiple recommendations for learning in statistics, including using real data and implementing the use of technology in classrooms. The use of technology to favor better learning is however not restricted to statistics, and teaching tools can be used in a wide variety of classes.

Creating simple (and preferably freely accessible) tools for instructors to make complex concepts easy to represent and to provide feedback concerning the students' knowledge development can help to bring a sense of presence amongst the students. In this concern, a large variety of teaching tools and approaches should be encouraged, alternating and modifying the way concepts are taught. As (Vaughn, 2009) proposed: "Another solution is found in innovative instructional paradigms in which the traditional lecture, with students passively listening, is replaced with more hands-on activities" (p.106). This could be achieved by creating small quizzes in class in which the students can participate anonymously. It could also be accomplished by running short surveys whose sub-scales would be dis- 
Table 1 - Comparison between three different interactivity tools

\begin{tabular}{|c|c|c|c|}
\hline \multirow[b]{2}{*}{ Characteristics } & \multicolumn{3}{|c|}{ Software } \\
\hline & ivote & Socrative & SurveyMonkey \\
\hline Does not require Login or account & Yes & No & No \\
\hline Is free & Yes & Yes & No * \\
\hline Contains item customizability & Yes & Yes & Yes \\
\hline Allows complete survey creation & No & No & Yes \\
\hline Presents the results visually & Yes $* *$ & Yes & Yes \\
\hline $\begin{array}{l}\text { Allows data exportation and is } \\
\text { compatible with other software }\end{array}$ & Yes $* * *$ & No & Yes \\
\hline Displays the questions & No & Yes & Yes \\
\hline Has Interactive games & No & Yes & No \\
\hline
\end{tabular}

Note. * Except for small, restricted use; ** Currently, display in PowerPoint for PC is supported; *** Currently, exports to Excel, SPSS, Mathematica, Python, and R are supported.

cussed in the class afterwards. Alternatively, data provided by the students could be fetched and analyzed in real time using statistical tools.

There is a wide range of teaching tools that can be used in a classroom. They can be loosely grouped into four categories:

1. Presentation tools: these allow the creation of presentations. An example of this category is PowerPoint from Microsoft, which is widely used and known for its simplicity and manipulability. It uses Visual Basic for Application (VBA) for programming (ivote's ability to display content inside the slides, discussed later, is based on VBA). An alternative is Prezi Inc (2016), offering an interactive display with moving effects and zooming transitions between slides.

2. Content providing tools: these tools provide information repositories accessible from a distance. These tools include Blackboard, commonly used to share information and documents in universities, but also YouTube, Dailymotion, streams, etc.

3. Exercise and simulation tools: these are used to present cases or simulations that the students need to handle as if it was a real-life situation. In statistics, for example, they can be used for the creation of data. Some tools like GRD (Harding \& Cousineau, 2014, 2015) and GSD (Harding \& Cousineau, 2016) can generate random data and samples within SPSS.

4. Interactivity tools: these are meant to incite the learner to participate in the learning process as a direct actor, which in return may influence motivation. They can be further divided in subcategories: (a) Blogs and chat rooms allow learners to discuss their questions; (b) Televoting tools allow learners to participate by answering to interactive questions. Good examples of these tools include Socrative, Virtual Tutor and clickers which will be addressed later in the text. ivote, the focus of this document, is also part of this category; (c) Survey tools are similar to televoting tools, but they are composed of multiple questions and are usually longer to complete. SurveyMonkey (SurveyMonkey, 1999) and Google Form are commonly used tools from this category, allowing the creation of full length surveys.

Other categories exist, depending on the different needs that emerge from the community. Also, some tools merge many functions in one single tool (e.g. Virtual Tutor offering presentation and televoting functions, or Blackboard offering documents, grades statistics and chat rooms).

One type of tool that we will discuss more in depth in this article is televoting. Televoting effectively gets the student to participate in his or her learning experience, counteracting the boredom associated with repetitive teaching methods if these are alternated on a regular basis. Most university students have a computer or a smartphone, which can be used to answer. The simpler the procedure, the faster the students will answer, and in larger numbers.

This software is effective when students are intimidated by the presence of their classmates and do not want to publicly show their opinion, in fear of social pressures. As seen in our classes, students participate in larger numbers when the choices are made anonymously, since it allows the participant to avoid peer pressure. These ideas motivated the creation of ivote.

\section{ivote}

ivote is a voting system for class polls and questions. It distinguishes itself from other software by being free and not requiring any registration. Its simplicity and versatility makes it useful for quick and efficient use in classrooms, meetings and conversation groups. The application is compatible with PowerPoint, Excel, SPSS, R, Python, etc. The results can therefore easily be fetched, displayed, trans- 
Figure 1 - The home page of ivote. It contains four fields for "Defining a new question”.

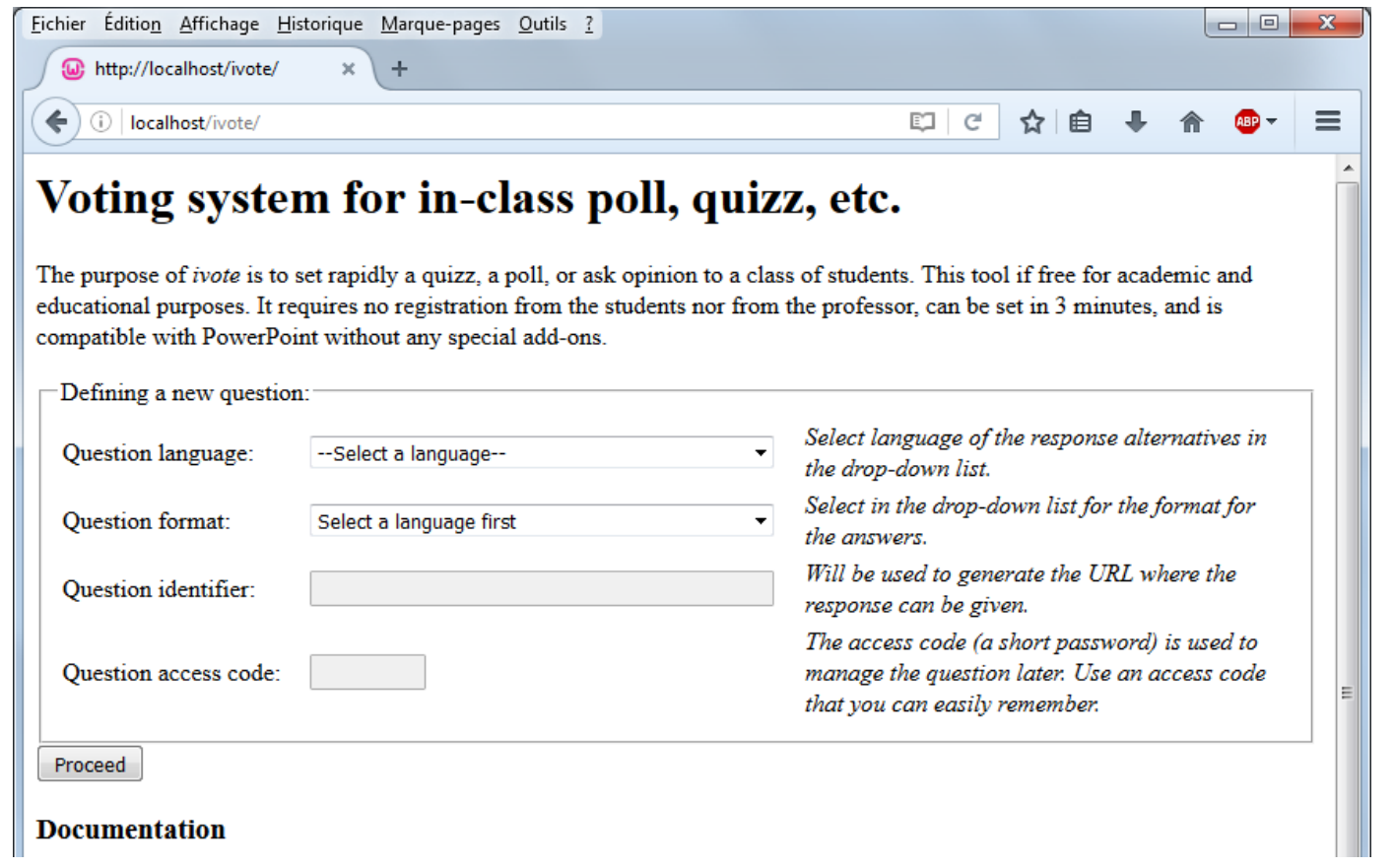

formed and analyzed. The software is accessible online, via www.tqmp.org/ivote.

It is a free project and it has been conceptualized to help instructors create new means of teaching by giving feedback concerning students' comprehension of the taught matter.

Alternatives to ivote are often not free resources. While some offer the possibility to create free accounts, registration and an email address are required to create these accounts; these free accounts often have limited functions compared to a paying account. This is the case of software like SurveyMonkey, which allows the exportation of data into SPSS, Excel and others (like ivote), but requires payment in order to be used at its full potential. The free part of the software is restricted to 10 questions and 100 respondents and export functions are disabled.

On the other hand, software like Socrative (Socrative, 2016) is free. It is simple and easy to understand; its interface makes it user-friendly. It allows the development of multiple choice questions, true or false, and short answer questions. Socrative also has functionalities, such as the space race game in which the student needs to answer to the most questions as fast as possible. It allows the development of full length questionnaires, with different types of questions and question randomization. However, data exportation and transformation is not available with
Socrative.

Table 1 summarizes the key aspects of these televoting software.

One of the major advantages ivote has compared to other software is its ability to display results in a PowerPoint presentation. It is achieved by using a macro, programmed with the Visual Basic for Applications (VBA) language. The manipulations will be explained in an upcoming section.

The alternatives to ivote usually require registering to the tool's website and, if used with a mobile device, downloading the application. Regarding ivote, students are not required to sign in, and the questions can be answered through the web on a mobile device. A Quick Response code (QR Code) can also be used to answer a question (however, to use the QR codes, a free application is required; an example of QR code is seen in Figure 3). The vote results can be shown, as mentioned earlier, in a PowerPoint presentation without leaving the slides. This means that the students' participation can be dynamic throughout the class, as the presentation does not need to be closed or minimized to use alternative resources. ivote is also not demanding in terms of bandwidth, which means it is faster, including on computers with slow Internet connections or outdated hardware. It automatically generates code for integration in many software environments. 
Figure 2 - Illustration of four types of formats. In panel a) 5-choice multiple choice format; panel b) free text format; panel c) Likert scale format; panel d) true or false format. Other formats also exist, as seen in Figure 1, bottom.

(a)

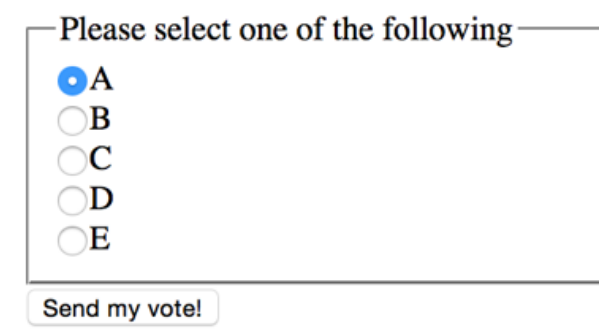

(c)

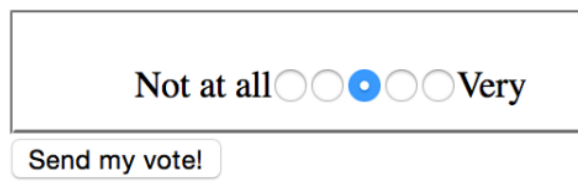

\section{How to use ivote}

To use ivote, three steps are required:

1- Create a question Go to www.tqmp.org/ivote (Figure 1 shows the content of the web page). Fill the four fields under "Defining a new question".

- The first field, Question language, allows the user to select the language in which the vote alternatives will be shown to the respondent. The choices presently available are French and English. Note that the website is in English only.

- The second field, Question format, allows the user to select in a drop-down list the format that is going to be used for the answers. It can be multiple-choice question items (four or five choices), true or false and yes or no. ivote also offers free text answers, integer number answers and Likert scale items as well.

- The third, Question identifier, is used to generate the URL that the respondent has to access to enter their responses.

- The fourth and last field, Question access code, defines a short password used to manage the question later, at the creator's discretion. It is recommended to use an easily remembered password. Note that this password will be added as an extension to the question's URL, so there is no need to remember the password if the management web page URL is saved in your bookmarks. When done, click Proceed. Two links should appear, (b)

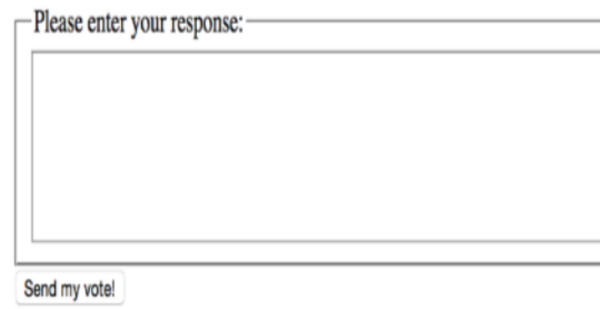

(d)

\begin{tabular}{l}
$\begin{array}{l}\text { Please select one of the following - } \\
\text { True } \\
\text { False }\end{array}$ \\
\hline Send my vote!
\end{tabular}

one "For the students" and one "For the instructor". The first link is used by students, to answer the questions. The second one is used to manage, complement and see a Quick-access dashboard.

As an example, if you created a new question that you named "myquestion" with the access code "d", then the following URL would be created to enter votes:

http: //www.tqmp.org/ivote/myquestion.php and this one to manage your question:

http://www.tqmp.org/ivote/myquestion.php ?Access Code $=\mathrm{d}$.

Both URLs begin the same (www.tqmp.org/ivote is the website hosting ivote), followed by the question name and the extension .php. The second adds an additional information, the access code. The access code should not be communicated to the students, as with this access code, the question can be changed or the votes erased. One possible naming scheme is to use the class code (e.g. psy101) followed by a question number (e.g., q1). Note that the question name is case sensitive, such that psy101q1 and psy101Q1 are not the same question name. The access code can be any string (also case sensitive). An instructor can use the same access code for all his or her questions. If lost, it is not possible to recover the access code.

The Quantitative Methods for Psychology 
Figure 3 - The content of the web page for the instructor. It shows in the top part (the "Quick-access dashboard") available information, including the URL and the QR code to distribute to the student; in the middle section ("Web-based access to some functions") links to some functions of ivote; in a third section not shown at the bottom of this one, it provides personalized code for PowerPoint, SPSS, etc. The question identifier used was "121212" and the access code was "121212" as well.

Quick-access dashboard:

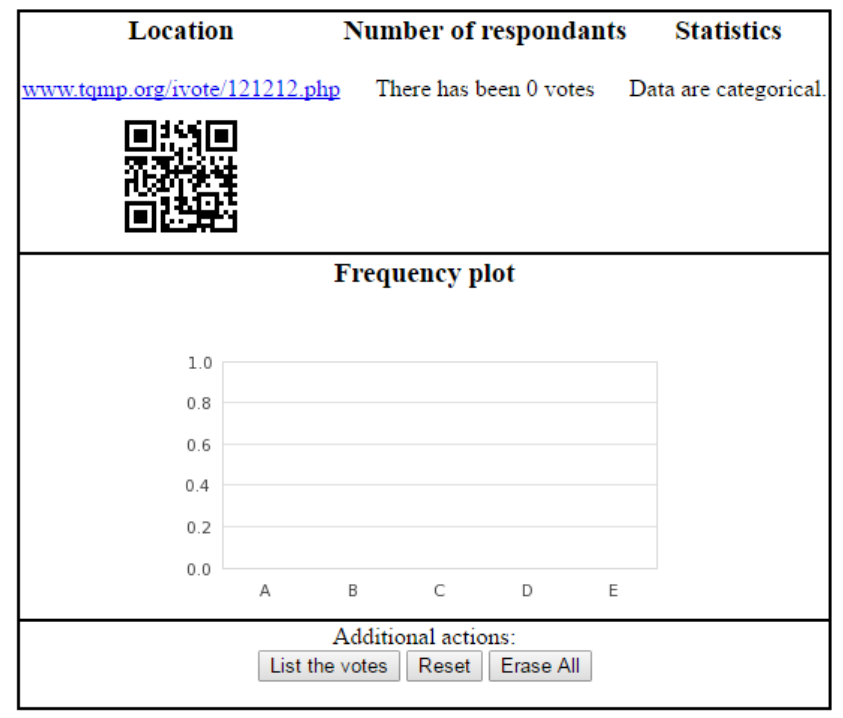

Web-based access to some functions:

- go to http://www.tqmp.org/ivote/121212.php?AccessCode=121212\&PlotOnly to get the vote results only

- go to http://www.tamp.org/ivote/121212.php?AccessCode=121212\&StatsOnly to get stats on the votes only

- go to http://www.tgmp.org/ivote/121212.php?AccessCode=121212\&NOnly to see the number of votes only

- go to http://www.tqmp.org/ivote/121212.php?AccessCode=121212\&URLOnly to see the question URL only

- go to http://www.tqmp.org/ivote/121212.php?AccessCode=121212\&QRCodeOnly to see a QRcode to this question

- go to http://www.tqmp.org/ivote/121212.php?AccessCode=121212\&DataOnly to see the votes in plain text

- go to http://www.tqmp.org/ivote/121212.php?AccessCode=121212\&Reset to reset the question

- go to http://www.tqmp.org/ivote/121212.php?AccessCode=121212\&EraseAll to erase the question and all the data

2- Use the question. The participant, in order to cast his or her vote, needs to go through these steps:

- Type the URL given by the instructor (see the previous section) on an Internet browser, or scan the QR code if the instructor displays it.

- Answer the question. The choice alternatives depend on which format was selected at creation. Figure 2 shows a few possible layouts. The results are recorded upon pressing "send my vote!"

- The votes can then be accessed by the instructor in the management section (next sub-section), displayed in PowerPoint (next section) or uploaded to a programming environment of your choice (how to do this with SPSS is illustrated in the last section).

ivote does not record IP addresses and does not use "cookies". Hence, it is impossible to track who made a vote. For the same reason, it is possible for a respondent to vote multiple times.

3- Manage the question: The instructor can manipulate the question by opening the management page through the specific URL address. The page is composed of three sections.

In the first section, how to access the question is recapitulated (URL link or QR code is available from this link; if the students have a QR code reader application on their smartphone, they can reach the URL faster). Some statistics such as the total number of votes regarding the current poll are displayed as well as a frequency plot.

In the second section, links are presented to activate the functions of ivote. There are functions to reset the question, if it needs to be used in a different class. The erase button is also useful if the creator wants to get rid of a question entirely. Figure 3 shows the first two sections of that page. 
In the third section, personalized code necessary for functions in Powerpoint, SPSS, R, Python and Mathematica are provided. Additional explanations concerning PowerPoint and SPSS are given in the next sections.

All data created for the test items are saved on the website, after the test creator gave a name and access code to its question. The votes can be shown directly in a frequency plot, or uploaded to a software of your choice. If the creator returns to the management page, it is possible that an outdated frequency plot is shown (as the image is stored in a cache memory on the local computer). Use the "refresh" button to regenerate the plot.

\section{Using ivote in PowerPoint}

It is not necessary to go to the management page to see the votes. It is possible to display the frequency plot directly in presentation software such as PowerPoint using VBA. That way, the instructor does not have to exit PowerPoint during the class. Displaying the results on a button click can be useful to show the answers to a class. It also allows providing feedback faster, since the instructor does not need to leave the presentation at any point. A button on the PowerPoint slide and a macro is required. Once the button is created on a slide, the instructor must double-click the button to open the VBA editor, copy/paste the code provided on the management page of ivote, and then close the VBA editor. This code is personalized to the question's name and access code; Listing 1 shows such code for a question called "myquestion" whose access code is "1212". This step can be done at any time before the class begins. Note that the PowerPoint document now contains a macro (i.e., VBA code) and must be saved as a .pptm document (PowerPoint document with macro). Upon opening the document, it might be necessary to answer "yes" to the query to activate the macros, as security features of Microsoft Office sometimes deactivate macros in such documents.

By default, the VBA code shows a frequency plot of the votes. It is possible to change function by replacing the "PlotOnly" command in the instruction by another command, as listed in the instructor's management page. One useful function is "NOnly", which shows how many votes have been received. The instructor can for example decide to wait until a certain number of votes have been received.

To create a button, it is necessary to use the developer tab. If not visible, it can be activated by going in the "File: Option" where, in "Personalize the ribbon", you can check the "Developer" tab. This step must be done only once for a given computer.

In the Developer tab, there is a "Command button" tool in the "controls" section. On any slide, create a button with this tool. From there, the instructor can rename the button and add the VBA code into the editor by double click- ing on the button. It is necessary to be connected to Internet for the macros to work. For further instructions concerning the steps to use this code within PowerPoint, see http://www.tqmp.org/ivote/iVoteWithPowerPoint.pptm.

Figure 4 shows an example of a slide with a button, and the frequency plot displayed after the button has been used.

\section{Using ivote in SPSS}

It is also possible to upload the votes to a data analysis software such as SPSS. The data can be uploaded to the software and then used to run any analysis provided to the students just minutes earlier. To that end, the instructor needs to copy and paste code similar to the one given in Listing 2 into the SPSS's syntax editor. Prior to this, an SPSS extension command (getivote.spe, available at www.tqmp.org/ivote/GETIVOTES.spe) must be installed once on a given computer (in SPSS, go to menu Tools: Extension bundles: Install extension bundle).

The user runs the whole command and the results are uploaded to SPSS's data editor. Just as with PowerPoint, an Internet connection is necessary. For further instructions concerning the steps to use this code within SPSS, see http://www.tqmp.org/ivote/iVoteWithSPSS.pptx

\section{ivote with other environments}

ivote can also be used in many other environments. For example, code for Python, Mathematica and R are also provided on the management page. The data can also be exported in other software, for example Excel. ivote is in fact compatible with any environment that can read web pages, as for example, Matlab, SAS, etc.

\section{Limitations of ivote}

The software comprises some limitations. First, all data and questions are erased after seven days. This is done to avoid the server to be overloaded with data from too many questions. Second, no more than 500 votes can be recorded for one question. Third, since no cookie is left by ivote, a student can answer more than once should he or she refresh the page. Fourth, the text of the question is not shown on the web page. The student only sees the choice alternatives; the text of the question must be shown by the instructor via PowerPoint or any other mean. Fifth, the display of results is static; if new votes have been recorded, the graphic must be refreshed (by, for example, running the macro again). Lastly, the ivote webpage display can be used on any operating system, but the PowerPoint macro is not compatible with Mac OS. 
Listing 1 a VBA script generated by ivote to be copied into PowerPoint. It is personalized to a question named "myquestion" with access code " 1212 ". In this code, the left and top positions are in pixels (-1 represents the center of the screen); the width and height are also in pixels (-1 represents the default size of the plot created by ivote)

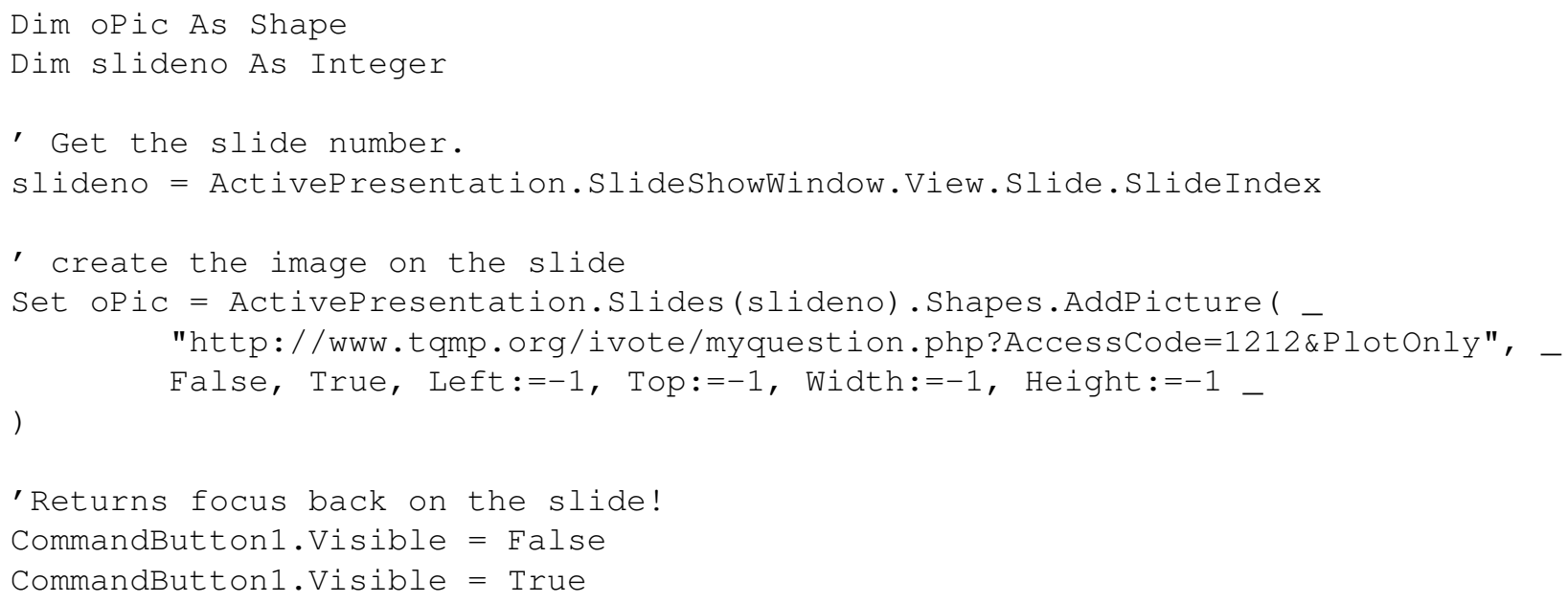

Listing 2 - SPSS Syntax generated by ivote to be copied in the Syntax window of SPSS. It is personalized for a question named "myquestion" with access code "1212". Executing the command will upload the votes into the current data editor

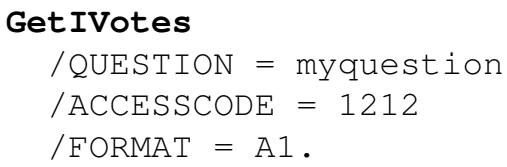

\section{Conclusion}

ivote is a simple and free software which has been created to help instructors diversify and enrich class presentations and activities. It allows student to be active in the class, using new technology, but at the same time remain anonymous. The instructor can then provide near immediate feedback and engage the students in discussion on the errors. It can be combined with presentation tools for optimal display and with data analysis tools, but can also be kept minimal if required. Compared to other resources, ivote possesses some advantages and some disadvantages, depending on the user's needs and personal preferences. The use of such a resource can have a meaningful impact on the students' construction of ideas, and can help to create a class dynamic where students engage in frequent small quizzes and are not afraid to commit to a response. From there, the instructor can start discussions, changing the class dynamic to a more positive one.

\section{Authors' note}

We would like to thank Marc-André Goulet, Bradley Harding and Vincent LeBlanc for their comments on an earlier version of this text. This research was supported in part by the Conseil pour la Recherche en Sciences Naturelles et en Génie du Canada by a scholarship to the first author and a research grant to the second author.

\section{References}

Aliaga, M., Cobb, G., Cuff, C., Garfield, J., Gould, R., Lock, R., ... Witmer, J. (2005). The gaise (guidelines for assessment and instruction in statistical education) college report. Alexandria,VA: The American Statistical Association.

Harding, B. \& Cousineau, D. (2014). Grd: an spss extension command for generating random data. The Quantitative Methods for Psychology, 10(2), 80-94.

Harding, B. \& Cousineau, D. (2015). Grd 2.0: an extended spss extension command for generating random data. The Quantitative Methods for Psychology, 11(3), 127138.

Harding, B. \& Cousineau, D. (2016). Gsd: an spss extension command for sub-sampling and bootstrapping datasets. The Quantitative Methods for Psychology, 12(2), 138-146. doi:10.20982/tqmp.12.2.p138

Hogg, R. V. (1991). Statistical education: improvements are badly needed. The American Statistician, 45, 342-343. 
Figure 4 ॥ An example of a slide containing a question, a button to display the vote results, and the frequency plot generated by ivote, obtained after the button has been used. The "Left" and "Top" attributes has been set to "Left:=240, Top:=330" so that the plot appears approximately next to the button.

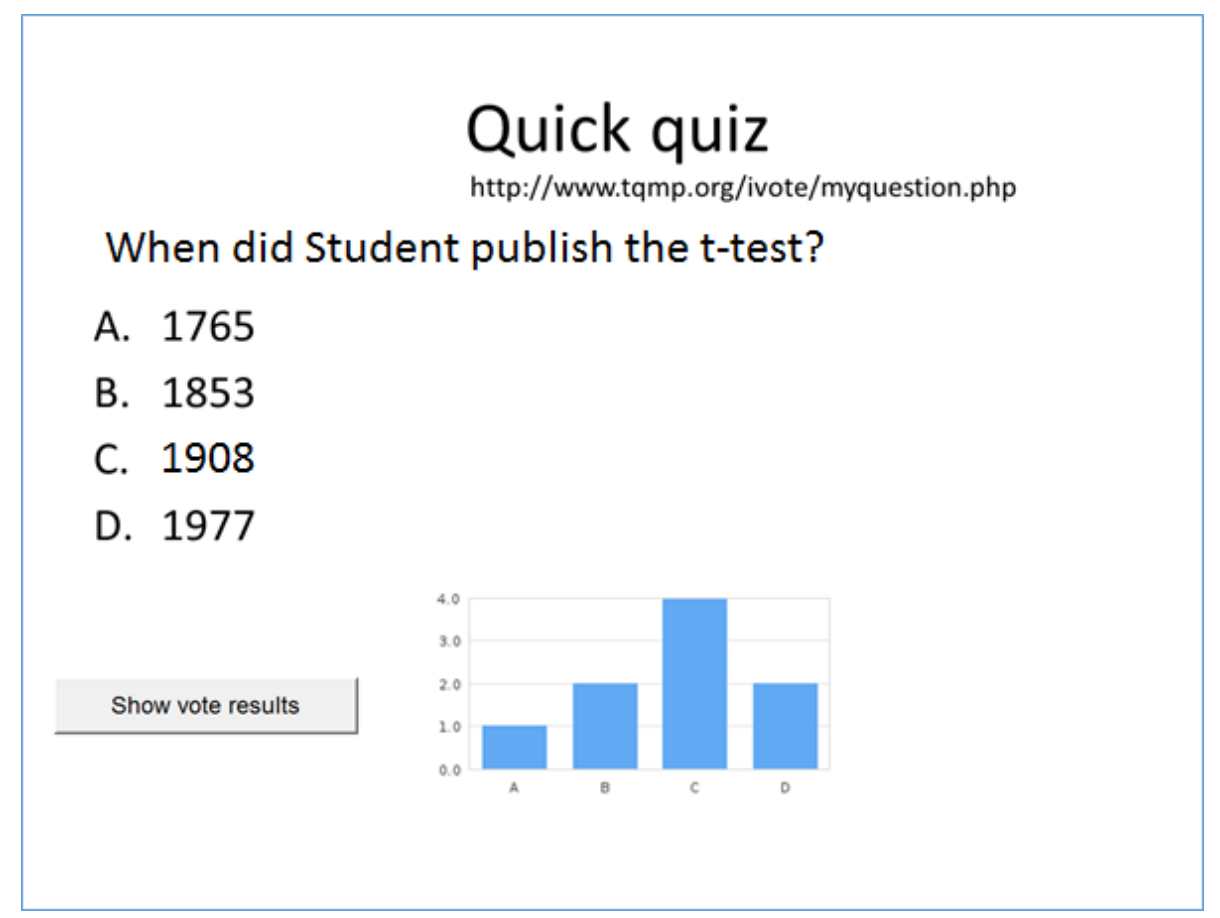

Prezi Inc. (2016). Prezi: the presentation software for when it matters (Version 1.0). Retrieved from http://prezi. com

Socrative. (2016). Socrative by mastery connect (Version 1.0). Retrieved from http://socrative.com

Stuart, M. (1995). Changing the teaching of statistics. The Statistician, 44(1), 45-54.
SurveyMonkey. (1999). Surveymonkey: free online survey software \& questionnaire tool (Version 1.0). Retrieved from https://www. surveymonkey.com/?ut_source= header

Vaughn, B. K. (2009). An empirical consideration of a balanced amalgamation of learning strategies in graduate introductory statistics classes. Statistics Education Research, 8(1), 106-130.

\section{Open practices}

- The Open Material badge was earned because supplementary material(s) are available on the journal's web site.

\section{Citation}

T. Groulx, J. \& Cousineau, D. (2016). Ivote: a simple system to conduct polls and quiz in class settings. The Quantitative Methods for Psychology, 13(1), 57-64. doi:10.20982/tqmp.13.1.p057

Copyright ( 2016, T. Groulx and Cousineau. This is an open-access article distributed under the terms of the Creative Commons Attribution License (CC BY). The use, distribution or reproduction in other forums is permitted, provided the original author(s) or licensor are credited and that the original publication in this journal is cited, in accordance with accepted academic practice. No use, distribution or reproduction is permitted which does not comply with these terms.

Received: 08/07/2016 Accepted: 18/10/2016 